\title{
Targeting the CXCL12/CXCR4 axis in acute myeloid leukemia: from bench to bedside
}

\author{
Byung-Sik Cho ${ }^{1,2}$, Hee-Je Kim ${ }^{1}$, and Marina Konopleva ${ }^{2}$
}

\begin{abstract}
${ }^{1}$ Department of Hematology, Catholic Blood and Marrow Transplantation Center, Seoul St. Mary's Hospital, Leukemia Research Institute, College of Medicine, The Catholic University of Korea, Seoul, Korea; ${ }^{2}$ Section of Molecular Hematology and Therapy, Department of Leukemia, The University of Texas MD Anderson Cancer Center, Houston, TX, USA
\end{abstract}

Received: July 25, 2016 Accepted: February 8, 2017

\section{Correspondence to}

Marina Konopleva, M.D. Section of Molecular Hematology and Therapy, Department of Leukemia, The University of Texas MD Anderson Cancer Center, 1515 Holcombe Blvd, Unit 0448, Houston, TX, 77030, USA

Tel: +1-713-794-1628

Fax: +1-713-745-4612

E-mail: mkonople@mdanderson.org
The interactions between the cancerous cells of acute myeloid leukemia (AML) and the bone marrow (BM) microenvironment have been postulated to be important for resistance to chemotherapy and disease relapse in AML. The chemokine receptor CXC chemokine receptor 4 (CXCR4) and its ligand, CXC motifligand 12 (CXCL12), also known as stromal cell-derived factor 1a, are key mediators of this interaction. CXCL12 is produced by the BM microenvironment, binds and activates its cognate receptor CXCR4 on leukemic cells, facilitates leukemia cell trafficking and homing in the BM microenvironment, and keeps leukemic cells in close contact with the stromal cells and extracellular matrix that constitutively generate growth-promoting and anti-apoptotic signals. Indeed, a high level of CXCR4 expression on AML blasts is known to be associated with poor prognosis. Recent preclinical and clinical studies have revealed the safety and potential clinical utility of targeting the CXCL12/CXCR4 axis in AML with different classes of drugs, including small molecules, peptides, and monoclonal antibodies. In this review, we describe recent evidence of targeting these leukemia-stroma interactions, focusing on the CXCL12/CXCR4 axis. Related early phase clinical studies will be also introduced.

Keywords: Leukemia, myeloid, acute; CXCR4; CXCL12

\section{INTRODUCTION}

Acute myeloid leukemia (AML) is a heterogeneous group of diseases characterized by the uncontrolled proliferation of hematopoietic stem cells (HSCs) and progenitors with a reduced capacity to differentiate into mature cells. Despite initial sensitivity to chemotherapy, long-term outcomes of AML patients remain poor and the majority eventually relapse [1]. The main challenge in improving outcomes of AML treatment is the development of strategies to overcome resistance to chemotherapy, but critical factors regulating the survival of resistant leukemic cells remain unknown. Some research has helped clarify the molecular abnormalities that underlie leukemogenesis in conjunction with clonal het- erogeneity, which can be facilitated by chemotherapy $[2,3]$. While genomic and epigenetic cell intrinsic mechanisms of drug resistance are rapidly being uncovered and targeted, with prime examples being inhibitors of oncogenic kinases (e.g., mutations of Fms-like tyrosine kinase-3 [FLT3]) and metabolic inhibitors of isocitrate dehydrogenase type 1 and 2, leukemic cells are also known to be strongly dependent upon their microenvironment in the bone marrow (BM) or stem cell 'niche.' It has been well established that BM stromal cells convey drug resistance in AML [4,5]. For leukemia-stroma interactions, the homing and adhesion of AML cells to the $\mathrm{BM}$ niche is critical. Many of the adhesion molecules, chemokine receptors, and signaling cascades that are critical to the homing of HSCs, maintenance, and egress 
from the BM niche are shared by the leukemic cells [6]. In this review, we describe recent evidence for targeting the leukemia-stroma interaction, focusing on the CXC motif ligand 12 (CXCL12)/CXC chemokine receptor 4 (CXCR4) axis. Related clinical studies will be also introduced, even though they were limited to the early phase of treatment.

\section{CXCL12-CXCR4 AXIS IN AML}

The CXCL12-CXCR4 axis is the key mediator of migration and homing of HSCs to the BM [7]. CXCL12 is a homeostatic chemokine, also known as stromal-derived factor $1 \mathrm{a}$, which is produced by the marrow stroma and epithelial cells. The major function is to regulate hematopoietic cell trafficking and secondary lymphoid tissue architecture [8]. CXCL12 binds and initiates signaling through its cognate receptors $\mathrm{CXCR}_{4}$ and $\mathrm{CXCR}_{7}$ (G-protein coupled chemokine receptors). CXCL12 is a specific ligand for $\mathrm{CXCR}_{4}$, whereas $\mathrm{CXCR}_{7}$ can bind both CXCL12 and CXCL11. CXCR4 is widely expressed on hematopoietic cells including HSCs, T cells, B cells, monocytes, macrophages, neutrophils, and eosinophils. Furthermore, the receptor is expressed in the brain, lung, colon, heart, kidney, and liver, as well as endothelial and epithelial cells, microglia, astrocytes and neuronal cells, and progenitor cells including endothelial and smooth muscle progenitors [8]. The pleiotropic activity of the CXCL12 pathway is critical for hematopoietic, neural, vascular, and craniofacial organogenesis and cardiac development [9]. Activation of CXCR4 and/or CXCR7 signaling may affect several major signaling pathways related to cell survival, proliferation, and migration [8]. The binding of CXCL12 to CXCR4 activates down-stream signals, resulting in the homing of HSCs to the marrow in response to CXCL12 gradients, quiescence and survival of HSCs, and regulation of the size of the HSC pool [7,10]. The CXCL12-CXCR4 axis is exploited by AML cells; whereby, it activates multiple prosurvival signaling pathways including the phosphoinositide 3-kinase $\left(\mathrm{PI}_{3} \mathrm{~K}\right) / \mathrm{AKT}$ and mitogen-activated protein kinase kinase (MAPK)/extracellular signal-regulated kinase (ERK) axes, and the YY1-mediated transcriptional activation of myelocytomatosis and B-cell lymphoma-extra large (BCL$\mathrm{XL})$ through the downregulation of microRNA let-7a
$[11,12]$.

Mohle et al. [13] first reported variable cell surface expression of CXCR4 in AML, which is functionally active with a positive correlation between the surface density of CXCR4 and CXCL12-induced migration. The preferential CXCL12-induced migration of immature leukemic cells that express $\mathrm{CD}_{34}, \mathrm{CD}_{3} 8$, and human leukocyte antigen-DR, and of marrow-derived cells compared to peripheral blood-derived cells, suggests that the CXCL12-CXCR4 axis plays a role in the anchoring of immature, quiescent leukemic cells in the BM [14]. Several groups have demonstrated the prognostic significance of CXCR4 expression in AML with or without the $\mathrm{FLT}_{3} /$ internal tandem duplication (ITD)-mutation [15-17], in which CXCR4 expression is associated with poor prognosis. A polymorphism in the CXCL12 coding gene (CXCL12 G801A) was shown to be associated with the clinical presentation of AML and a higher frequency of extramedullary leukemic infiltration [18]. Importantly, Fiegl et al. [19] demonstrated that surface CXCR4 expression was upregulated and migration toward CXCL12 was enhanced under hypoxic conditions, suggesting that the level of CXCR4 surface expression may change under physiological conditions of hypoxic BM. Together, these data strongly indicate that disruption of the CXCL12-CXCR4 axis may influence the fate of AML cells. Furthermore, Sison et al. [20] recently showed that AML cell lines variably upregulated CXCR4 upon chemotherapy treatment, resulting in increased CXCL12-mediated chemotaxis and a stromal-mediated survival advantage in pediatric AML, suggesting upregulation of surface CXCR4 as one of the mechanisms of chemotherapy resistance. Thus, a therapeutic strategy targeting the CXCL12-CXCR4 axis may represent an attractive investigational approach to disrupt the leukemia-stromal interaction of AML.

\section{CXCR4 INHIBITORS: PRECLINICAL DATA}

\section{Small molecules}

Tavor et al. [21] reported the importance of the CXCL12-CXCR4 axis in the regulation of in vivo motility and development of human AML stem cells and identified CXCR4 neutralization as a potential treatment for AML. They found that all AML cells tested expressed internal 
$\mathrm{CXCR}_{4}$ and CXCL12, even cells without surface CXCR4 expression, and observed an antileukemia effect of the in vivo CXCR4 neutralization by blocking antibody in an AML xenograft model. Importantly, CXCR4 inhibition did not significantly affect the engraftment of normal human progenitors into nonobese diabetic (NOD)/severe combined immunodeficiency (SCID) mice. Subsequently, several groups explored whether the US Food and Drug Administration (FDA)-approved small molecular CXCR4 inhibitor, plerixafor (AML3100), affected the trafficking and survival of AML cells in vitro and in vivo. AMD3100 caused inhibition of the CXCL12-induced migration of AML blasts, an outgrowth of leukemia colony forming units, and a promigratory effect of $\mathrm{CXCL}_{12}$ on AML engraftment into NOD/SCID mice [22]. Another study also demonstrated that AMD3100 inhibited proliferation in AML cell lines and triggered changes that mimicked differentiation [23].

In 2009, two preclinical studies evaluated the efficacy of two small molecular CXCR4 inhibitors, AMD310o and $\mathrm{AMD}_{3465}$, in combination with chemotherapy or targeted agents, in human AML xenograft models. These studies demonstrated that these CXCR4 inhibitors exerted chemosensitizing effects, which supported the notion that the CXCL12-CXCR4 axis plays a key role in chemoresistance via leukemia-stromal interactions [4,5]. Zeng et al. [5] reported that CXCR4 inhibition by $\mathrm{AMD}_{3465}$, a derivative of $\mathrm{AMD}_{3100}$, increased the sensitivity of $\mathrm{FLT}_{3}$ inhibitors sorafenib and cytarabine in xenograft models of mouse leukemic cells with or without an FLT3-mutation. $\mathrm{AMD}_{3465}$, alone or in combination with granulocyte colony-stimulating factors (G-CSF), induced the mobilization of AML cells and progenitor cells into the circulation and enhanced the antileukemia effects of cytarabine and sorafenib, resulting in a reduced leukemia burden and prolonged survival in mice. Further, CXCR4 inhibition antagonized the stroma or CXCL12-induced chemotaxis and CXCL12-induced activation of prosurvival signaling pathways, such as $\mathrm{PI}_{3} \mathrm{~K} / \mathrm{AKT}$ and MAPK/ ERK, in leukemic cells. In a separate report, Nervi et al. [4] showed the chemosensitizing effect of AMD3100 in acute promyelocyte leukemia (APL) mouse models with cytarabine, and the administration of AMD310o to leukemic mice induced an increase of leukemic cells in blood, which peaked at 3 hours and returned to baseline by 12 hours.
Chen et al. [12] exploited the novel mechanism of chemosensitization in AML, and identified a CXCR4/let-7a/ BCL-XL axis that may be responsible for the chemoresistance. They found that the overexpression of surface $\mathrm{CXCR}_{4}$ and activation of $\mathrm{CXCR}_{4}$ signaling results in downregulation of microRNA let-7a, which led to increased expression of the antiapoptotic protein BCL-XL in AML cells, suggesting that let-7a plays an important role in AML cell chemoresistance. This report extends the concept of targeting CXCR4 in AML by defining a complex network with the microRNA let-7a.

\section{Peptides}

As an alternative to small molecular CXCR4 inhibitors, many potent peptide $\mathrm{CXCR}_{4}$ inhibitors have been identified [24] and tested in AML models. In 2011, Beider et al. [25] demonstrated that a small peptide CXCR4 inhibitor, 4F-benzoyl-TN14003 (BKT140), exhibits CXCR4-dependent preferential cytotoxicity toward leukemia and multiple myeloma in contrast to $\mathrm{AMD}_{3100}$. They found that BKT140 stimulated cell death through apoptosis in an APL cell line (NB4), and significantly reduced the growth of a subcutaneous xenograft with $\mathrm{NB}_{4}$ cell lines in SCID/beige mice. Zhang et al. [11] also reported that BKT140 alone selectively induced leukemia regression in primary AML xenograft models when AML cells expressed high CXCR4 levels and displayed a significant migratory response to CXCL12. Additionally, CXCR4 inhibition caused increased apoptosis of leukemic cells in vitro and in vivo. Both studies suggest a potential therapeutic use for BKT140 as a monotherapy. In 2015, Cho et al. [26] also demonstrated remarkable antileukemia activity of peptidic CXCR4 inhibitor, LY2510924, as a monotherapy and in combination with anti-AML chemotherapy. In vitro data revealed that LY2510924 at nanomolar concentrations rapidly and durably disrupts the CXCL12-CXCR4 axis in AML cells, which inhibits proliferation of AML cells rather than causing cell death (in contrast to BKT140 data). Using primary AML xenograft models, they found that LY2510924 causes mobilization of leukemic cells into the circulatory system, inhibits multiple prosurvival signals generated by the CXCL12/CXCR4 axis, and induces myeloid differentiation; thereby, producing antileukemia effects as monotherapy. This antileukemia activity strongly synergized with chemotherapy consisting of cytarabine and doxo- 
rubicin in xenograft models, resembling standard induction chemotherapy in human trials. In summary, preclinical data of peptidic $\mathrm{CXCR}_{4}$ inhibitors suggest promising antileukemia effects as monotherapy in addition to their chemosensitization effects. However, because the findings vary, more research is needed to explore the potential for CXCR4 inhibitors to induce cell death through apoptosis.

\section{Monoclonal antibodies}

In recent years, several preclinical studies have reported promising antileukemia effects of anti-CXCR4 monoclonal antibodies as monotherapy. In contrast to small molecules and peptide CXCR4 inhibitors, monoclonal antibodies are expected to exert antileukemia effects through additional mechanisms, such as antibody-dependent cell-mediated cytotoxicity (ADCC) and/or complement-dependent cytotoxicity (CDC). In 2013, Kuhne et al. [27] introduced ulocuplumab (BMD-936564/MDX1338), a fully humanized immunoglobulin $\mathrm{G}_{4}$ (IgG4) monoclonal antibody that specifically recognizes human CXCR4. They found that ulocuplumab exhibits antitumor activity in established tumors including subcutaneous xenograft models of APL and induces apoptosis on a panel of cell lines including AML. They also proposed that antibody-induced apoptosis is one of the mechanisms of tumor-growth inhibition. Another humanized anti-CXCR4 IgG4 monoclonal antibody, LY2624587, also exhibited potential for inducing apoptosis in human lymphoma and leukemia in vitro and in vivo [28]. Preclinical data for the anti-CXCR4 IgG1 monoclonal antibody, PF-06747143, were recently presented at the Annual Meeting of the American Society of Hematology; the authors suggested that CDC and ADCC are mechanisms involved in the antileukemia effect in AML cell lines [29]. PF-06747143 exerted an antileukemia effect as monotherapy in primary AML xenograft models [30]. Overall, the preclinical data, as well as the plausible additional mechanisms for AML, suggest that anti-CXCR4 monoclonal antibodies have promise in clinical applications, while also raising concerns about toxicity in the process of normal hematopoiesis.

\section{PERSPECTIVES}

The preclinical data discussed above strongly suggest that the CXCL12/CXCR4 axis is a critical component of microenvironment-mediated drug resistance, which diminishes the activity of most cytotoxic drugs used in AML therapy and of tyrosine kinase inhibitors. Several different mechanisms of CXCR4 inhibition responsible for antileukemia effects have been identified: physical mobilization effects, decreased prosurvival signaling via CXCL12-CXCR4 downstream signaling (AKT and MAPK pathways), the induction of differentiation, effects on BCL-XL via the CXCR4/YY1/let-7a axis (even on non-mobilized AML cells), and the activation of ADCC and/or CDC in the case of anti-CXCR4 monoclonal antibodies. These mechanisms require further rigorous validation in clinical trials, and novel mechanisms of drug resistance mediated by the CXCL12/CXCR4 axis in AML need to be exploited.

To enhance the efficacy of CXCR4 inhibitors in disrupting the leukemia-stroma interaction, other adhesion molecules, such as CD44, very late antigen (VLA-4), or E-selectin on HSCs/AML cells, could be simultaneously blocked. Blocking CXCR4 and other adhesion molecules in parallel could theoretically eliminate the protection provided by the interaction of leukemic cells with their BM microenvironment. Preclinical data in murine models have demonstrated the effectiveness of targeting adhesion molecules, such as VLA-4, using blocking agents (natalizumab and $\mathrm{AS}_{101}$ ) [31,32], a small molecule inhibitor of E-selectin (GMI-1271) [33], or a CD44-specific monoclonal antibody [34] to dislodge leukemic cells from their protective BM niche. In particular, CD44 is an exciting target to mobilize leukemic cells out of their protective niche because leukemic stem cells (LSCs) have been found to be more dependent on $\mathrm{CD}_{44}$ for their anchoring in the BM niche than normal HSCs [34]. VLA-4 is also an emerging target for AML because vascular cell adhesion molecule 1 engagement of VLA-4 was found to mediate cross-talk between leukemia and stromal cells [35]. FDA-approved drugs that target CD 44 and VLA-4 for other diseases, such as bivatuzumab (an anti-CD44v6 monoclonal antibody), are currently being used for clinical trials of solid tumors, and natalizumab (an anti-VLA-4 monoclonal antibody) is being currently used to treat relapsing multiple sclerosis and 
inflammatory bowel disease. These medications could be used in conjunction with $\mathrm{CXCR}_{4}$ inhibitors to disrupt leukemia-stroma interactions more efficiently. An effective CXCR4 blockade, with or without the blockade of adhesion molecules, may translate into significant antileukemia activity in clinical applications. Indeed, some of these blockade mechanisms have advanced into clinical studies. However, the enhanced efficacy of a CXCR4 blockade has the potential to cause unwanted long-term effects on the maintenance of healthy HSCs. Furthermore, several studies have reported the function of $\mathrm{CXCL}_{2}$ and $\mathrm{CXCR}_{4}$ in multiple aspects of B- and T-cell development as well as in the regulation of effector immune responses [36], which could be affected by a CXCR4 blockade. Thus, the safety of a CXCR4 blockade in clinical applications should be carefully evaluated in combination therapeutic strategies with targeted agents and with conventional chemotherapy. For the design of clinical trials, the dosing schedule for combination therapy may be critical to the success of treatment. Additionally, Han et al. [37] reported that a CXCR4 blockade in combination with cytarabine may enhance the cytotoxicity of immune cells in a mouse AML model, suggesting that the effect of a CXCR4 blockade on immune cells in combination therapeutic strategies should be further evaluated.

Targeting the other part of the CXCL12-CXCR4 axis, the chemokine CXCL12, represents an attractive alternative approach. Recently, preclinical data on an RNA oligonucleotide in its L-configuration, known as the Spiegelmer NOX-A12 that binds and antagonizes CXCL12, revealed that NOX-A12 effectively interferes with chronic lymphocytic leukemia (CLL) cell migration and marrow stroma-mediated drug resistance [38]. After completion of a clinical phase 1 trial involving healthy volunteers [39], NOX-A12 is currently being tested in an ongoing phase 2 trial in patients with relapsed CLL and multiple myeloma.

\section{CXCR4 INHIBITORS: CLINICAL DATA}

\section{Small molecules}

Based on the encouraging preclinical evidence of CXCR4 inhibition in AML, a number of clinical studies involving different classes of agents have been initiated. How- ever, the evidence for safety and efficacy is currently limited to early-phase clinical trials (Table 1). Most related clinical trials have been designed to investigate the chemosensitizing effect of AML3100 in combination with chemotherapy or targeted agents. Uy et al. [40] reported a phase $1 / 2$ study of $A M D_{3100}$ in combination with chemotherapy in relapsed/refractory AML. In the study, 52 patients were treated with AMD3100 in combination with mitoxantrone, etoposide, and cytarabine (MEC). They demonstrated approximately a 2-fold mobilization of leukemic blasts into circulation, which returned to baseline within 12 hours. There was no evidence of symptomatic hyperleukocytosis or delayed count recovery, and a complete remission (CR) and CR with incomplete count recovery (CRi) rate of $46 \%$ was achieved, which is encouraging compared to published studies of MEC alone. In a randomized phase 3 study of MEC, the overall CR rate of MEC was $21 \%$ [41]. In a frontline setting, preliminary data from a phase 1 study of AMD3100 in combination with cytarabine and daunorubicin $(7+$ 3 regimen) revealed that the toxicity and hematopoietic recovery expected with this $7+3$ regimen was not significantly altered by the addition of AMD3100 [42]. The authors observed transient mobilization of AML blasts into circulation immediately following AMD3100 treatment, and achieved a CR/CRi of $76 \%$. A study of newly diagnosed, elderly patients with AML found that a combination of $\mathrm{AMD}_{3} 100$ with 10 days-decitabine regimen resulted in a $43 \% \mathrm{CR} / \mathrm{CRi}$; notably, $52 \%$ of patients without previous hypomethylating agent exposure and 53\% of patients with adverse cytogenetics achieved CR/CRi [43]. The authors administered AMD3100 concomitantly with decitabine in every other cycle, and the maximal tolerable dose used was $810 \mu \mathrm{g} / \mathrm{kg}$ without clinically significant hyperleukocytosis. Overall, these early-phase clinical data suggest that combining AMD3100 with chemotherapy or decitabine is safe and associated with favorable response rates as well as a transient mobilization of leukemic cells into circulation by AMD3100. Based on preclinical data indicating increased activity of sorafenib when combined with AMD310o and G-CSF in FLT3-ITD mutated AML [5], Andreeff et al. [44] completed a phase 1 study testing this finding in patients with FLT3-ITD mutated relapsed/refractory AML. The authors observed a 40-fold increase in circulating blasts as a result of mobilization, with preferential mobiliza- 
Table 1. Clinical trials of CXCR4 inhibitors in acute myeloid leukemia

\begin{tabular}{|c|c|c|c|c|c|}
\hline $\begin{array}{l}\text { CXCR4 } \\
\text { inhibitors }\end{array}$ & Combined regimens & Phase & Patient population & Sponsor & $\begin{array}{c}\text { ClinicalTrials.gov } \\
\text { identifier }\end{array}$ \\
\hline \multirow[t]{9}{*}{ Plerixafor } & $\begin{array}{l}\text { Mitoxantrone + Etoposide + } \\
\text { Cytarabine }\end{array}$ & $1 / 2$ & rrAML & Washington University & NCTo0512252 \\
\hline & $\begin{array}{l}\text { G-CSF + Mitoxantrone + } \\
\text { Etoposide + Cytarabine }\end{array}$ & $1 / 2$ & rrAML & Washington University & NCToo906945 \\
\hline & Decitabine & 1 & $\begin{array}{l}\text { Untreated } \\
\text { elderly AML }\end{array}$ & Cornell University & NCTo1352650 \\
\hline & G-CSF + Sorafenib & 1 & $\begin{array}{l}\text { rrAML with } \\
\text { FLT3-ITD }\end{array}$ & $\begin{array}{l}\text { M.D. Anderson Cancer } \\
\text { Center }\end{array}$ & NCToo943943 \\
\hline & Daunorubicin + Cytarabine & 1 & Untreated AML & Genzyme/Sanofi & NCTo0990054 \\
\hline & $\begin{array}{l}\text { Daunorubicin + Clofarabine } \\
\text { or Cytarabine }\end{array}$ & 1 & $\begin{array}{l}\text { Untreated elderly } \\
\text { AML }\end{array}$ & Cardiff University & NCTo1236144 \\
\hline & Cytarabine + Etoposide & 1 & rrAML & Emory & NCTo1319864 \\
\hline & Clofarabine & $1 / 2$ & $\begin{array}{l}\text { Untreated } \\
\text { elderly AML }\end{array}$ & $\begin{array}{l}\text { M.D. Anderson } \\
\text { Cancer Center }\end{array}$ & NCTo116o354 \\
\hline & $\begin{array}{l}\text { Fludarabine + Idarubicin + } \\
\text { Cytarabine + G-CSF }\end{array}$ & $1 / 2$ & rrAML & PETHEMA & NCTo1435343 \\
\hline \multirow[t]{2}{*}{ BL-8040 } & $\begin{array}{l}\text { Mitoxantrone + Etoposide + } \\
\text { Cytarabine }\end{array}$ & 1 & rrAML & BioLineRx & NCTo1838395 \\
\hline & Cytarabine as consolidation & 2 & AML in remission & Dr. Petra Tschanter & NCTo2502968 \\
\hline LY2510924 & Idarubicin + Cytarabine & 1 & rrAML & $\begin{array}{l}\text { M.D. Anderson } \\
\text { Cancer Center }\end{array}$ & NCTo2652871 \\
\hline \multirow[t]{2}{*}{$\begin{array}{l}\text { Ulocuplum- } \\
\text { ab }\end{array}$} & Low-dose cytarabine & 1 & $\begin{array}{l}\text { Untreated AML, } \\
\text { unfit for intensive } \\
\text { induction }\end{array}$ & Bristol-Myers Squibb & NCT02305563 \\
\hline & $\begin{array}{l}\text { Mitoxantrone + Etoposide + } \\
\text { Cytarabine }\end{array}$ & 1 & rrAML & Bristol-Myers Squibb & NCTo1120457 \\
\hline
\end{tabular}

rrAML, relapsed/refractory AML; G-CSF, granulocyte colony-stimulating factor; AML, acute myeloid leukemia; FLT3-ITD, Fms-like tyrosine kinsase-3 with internal tandem duplication mutation.

tion of leukemic versus non-leukemic cells. A CR/CRi of $28 \%$ and a partial response of $33 \%$ were achieved, and responses included three patients refractory to previous FLT3 inhibitors. Side effects included hyperleukocytosis, hypertension, hand foot syndrome, and diarrhea, the latter two attributable to sorafenib. This striking mobilization of leukemic cells by AMD3100/G-CSF provides proof of principle among FLT3-mutated AML patients, and the preferential mobilization of AML over normal cells further supports the clinical development of this treatment option, while the on-target side effects of hyperleukocytosis should be cautiously monitored.

\section{Peptides}

The peptidic CXCR4 inhibitor, BL-8040 (BKT140), has prolonged pharmacodynamic effects and exerts direct proapoptotic activity against AML cells $[11,25]$. In a phase $1 / 2$ trial of patients with relapsed/refractory AML, patients received 2 days of BL-8040 as a monotherapy followed by BL-8040 and cytarabine for 5 days [45]. The preliminary results of this study suggest that all tested doses of BL-8040 are safe. Two days of treatment with BL-8040 mobilized a 40.2-fold increase in immature AML progenitors from the marrow, followed by a $58 \%$ decrease in leukemia progenitor cells in the marrow on day 3. Apoptosis induction of blasts was detected in 5/9 samples available for analysis, which was associated with a 3.1-fold increase in monocytic and granulocytic terminal differentiation. During dose escalation in 22 patients, a CR/CRi of $38 \%$ was achieved. These findings 
suggest that the induction of apoptosis and differentiation, as well as mobilization of AML blasts from the protective marrow, may act as mechanisms of CXCR4 inhibition. Future combination studies with BL-8040 are ongoing or planned.

\section{Monoclonal antibodies}

Becker at al. [46] reported a phase 1 study using ulocuplumab (BMD-936564/MDX-1338), an anti-CXCR4 fully human $\mathrm{IgG}_{4}$ monoclonal antibody, which appears to have direct proapoptotic effects [27], in combination with MEC, in 73 patients with relapsed/refractory AML. Different doses of ulocuplumab were administered one week prior to starting MEC, and 3 additional weekly doses per MEC cycle thereafter, without dose-limiting toxicity. A fairly respectable CR/CRi of $51 \%$ was achieved, and, notably in four patients, CR/CRi was documented after a single dose of ulocuplumab monotherapy. Transient, mild, or moderate thrombocytopenia were the only treatment-related adverse events. A median 2- and 5 -fold mobilization of leukocytes and leukemic blasts into the circulation was reported at day 8 , respectively. This first clinical report of an anti-CXCR4 monoclonal antibody suggests that ulocuplumab exhibits safe and significant antileukemia activity in AML. The elimination of BM leukemia observed after a single dose of ulocuplumab in selected patients suggests a contribution from other mechanisms, such as ADCC, which may be related to the nature of the antibody. Future clinical trials with other antibody drugs, such as $\mathrm{LY}_{2} 624587$ (IgG4) and PF-06747143 (IgG1), are worth considering.

\section{Perspectives}

Early-phase trials suggest that the addition of CXCR4 inhibitors is tolerable and appears to exhibit signs of clinical activity. Further phase 2 and 3 trials are required to determine the benefit of CXCR4 inhibitors in combination with chemotherapy or targeted agents. Despite their small number, short follow-up, and small patient populations, early-phase trials indicate that CXCR4 inhibitors are generally safe, but they may lead to an on-target adverse event of hyperleukocytosis, which should be carefully considered in future patient selection, dosing, and schedule of combinations. Another potential adverse effect is an unwanted effect on healthy HSCs, such as myelosupression, or a deleterious effect on immune cells, even though currently available clinical data have not reported a significant delay in the recovery of blood cells after chemotherapy.

Most importantly, an optimal combination and schedule is required to enhance the efficacy of $\mathrm{CXCR}_{4}$ inhibitors without increasing potential toxicity. Different pharmacokinetic and pharmacodynamic characteristics of $\mathrm{CXCR}_{4}$ inhibitors should be considered to design clinical trials, particularly for drugs with a potential to directly induce cell death, such as BL-8040 or ulocuplumab.

The main subjects for ongoing clinical studies are patients with relapsed/refractory AML of any age and newly diagnosed, elderly patients with AML, the latter presenting a huge unmet need. Another unexplored area for future research is targeting AML-niche interactions in patients in remission after conventional chemotherapy to eradicate minimally residual disease or LSCs, which are thought to be responsible for the majority of relapses [47].

\section{CONCLUSIONS}

The significant heterogeneity and multi-clonal nature of AML, revealed by large-scale DNA sequencing studies, has been a barrier to improving treatment outcomes of drugs targeting deregulated signaling pathways. In particular, significant unmet needs remain in subjects without known driver mutations or for whom no drugs are currently available, as well as elderly AML patients who are unfit for intensive chemotherapy or curative transplantation approaches. As a solution, targeting the leukemia-stroma interaction is an attractive strategy to improve the outcome of AML in a non-cell autonomous fashion, which could be applied to a broad range of subjects regardless of underlying driver mutations. The lack of reliance on a particular driver mutation and the wide applicability to various disease types makes targeting the microenvironment a worthy endeavor. Among various potential targets to uncouple leukemic cells from their protective niche, agents interfering with the CXCL12/CXCR4 axis have been studied in several early-phase clinical trials. The results of selected combination trials, in particular with second-generation CXCR4 antagonists, appear to be promising and worth pursu- 
ing. Well-tolerated and highly efficacious CXCR4 inhibitors could be an ideal adjunct therapy alongside drugs directly targeting driver mutations to aim for curative treatment. Ongoing and future studies will elucidate the usefulness of targeting the CXCL12/CXCR4 axis in AML. Further, ongoing preclinical studies harnessing novel mechanisms of leukemia-stroma interactions will likely elucidate novel therapeutic targets in the near future.

\section{Conflict of interest}

No potential conflict of interest relevant to this article was reported.

\section{Acknowledgments}

This research was supported by the Basic Science Research Program through the National Research Foundation of Korea (NRF), funded by the Ministry of Science, ICT \& Future Planning (NRF-2015R1C1A1A01052764).

\section{REFERENCES}

1. Estey E, Dohner H. Acute myeloid leukaemia. Lancet 2006;368:1894-1907.

2. Ley TJ, Ding L, Walter MJ, et al. DNMT3A mutations in acute myeloid leukemia. N Engl J Med 2010;363:2424-2433.

3. Ding L, Ley TJ, Larson DE, et al. Clonal evolution in relapsed acute myeloid leukaemia revealed by whole-genome sequencing. Nature 2012;481:506-510.

4. Nervi B, Ramirez P, Rettig MP, et al. Chemosensitization of acute myeloid leukemia (AML) following mobilization by the CXCR4 antagonist AMD3100. Blood 2009;113:62066214 .

5. Zeng Z, Shi YX, Samudio IJ, et al. Targeting the leukemia microenvironment by CXCR4 inhibition overcomes resistance to kinase inhibitors and chemotherapy in AML. Blood 2009;113:6215-6224.

6. Peled A, Tavor S. Role of CXCR4 in the pathogenesis of acute myeloid leukemia. Theranostics 2013;3:34-39.

7. Sugiyama T, Kohara H, Noda M, Nagasawa T. Maintenance of the hematopoietic stem cell pool by CXCL12-CXCR4 chemokine signaling in bone marrow stromal cell niches. Immunity 2006;25:977-988.

8. Teicher BA, Fricker SP. CXCL12 (SDF-1)/CXCR4 pathway in cancer. Clin Cancer Res 2010;16:2927-2931.

9. Duda DG, Kozin SV, Kirkpatrick ND, Xu L, Fukumura D,
Jain RK. CXCL12 (SDF1alpha)-CXCR4/CXCR7 pathway inhibition: an emerging sensitizer for anticancer therapies? Clin Cancer Res 2011;17:2074-2080.

10. Greenbaum A, Hsu YM, Day RB, et al. CXCL12 in early mesenchymal progenitors is required for haematopoietic stem-cell maintenance. Nature 2013;495:227-230.

11. Zhang Y, Patel S, Abdelouahab H, et al. CXCR4 inhibitors selectively eliminate CXCR4-expressing human acute myeloid leukemia cells in NOG mouse model. Cell Death Dis 2012;3:e396.

12. Chen Y, Jacamo R, Konopleva M, Garzon R, Croce C, Andreeff M. CXCR4 downregulation of let-7a drives chemoresistance in acute myeloid leukemia. J Clin Invest 2013;123:2395-2407.

13. Mohle R, Bautz F, Rafii S, Moore MA, Brugger W, Kanz L. The chemokine receptor CXCR-4 is expressed on $\mathrm{CD}_{34+}$ hematopoietic progenitors and leukemic cells and mediates transendothelial migration induced by stromal cell-derived factor-1. Blood 1998;91:4523-4530.

14. Voermans C, van Heese WP, de Jong I, Gerritsen WR, van Der Schoot CE. Migratory behavior of leukemic cells from acute myeloid leukemia patients. Leukemia 2002;16:650-657.

15. Rombouts EJ, Pavic B, Lowenberg B, Ploemacher RE. Relation between CXCR-4 expression, Flt3 mutations, and unfavorable prognosis of adult acute myeloid leukemia. Blood 2004;104:550-557.

16. Konoplev S, Rassidakis GZ, Estey E, et al. Overexpression of CXCR4 predicts adverse overall and event-free survival in patients with unmutated $\mathrm{FLT}_{3}$ acute myeloid leukemia with normal karyotype. Cancer 2007;109:1152-1156.

17. Spoo AC, Lubbert M, Wierda WG, Burger JA. CXCR4 is a prognostic marker in acute myelogenous leukemia. Blood 2007;109:786-791.

18. Dommange F, Cartron G, Espanel C, et al. CXCL12 polymorphism and malignant cell dissemination/tissue infiltration in acute myeloid leukemia. FASEB J 2006;20:19131915 .

19. Fiegl M, Samudio I, Clise-Dwyer K, Burks JK, Mnjoyan Z, Andreeff M. CXCR4 expression and biologic activity in acute myeloid leukemia are dependent on oxygen partial pressure. Blood 2009;113:1504-1512.

20. Sison EA, McIntyre E, Magoon D, Brown P. Dynamic chemotherapy-induced upregulation of CXCR4 expression: a mechanism of therapeutic resistance in pediatric AML. Mol Cancer Res 2013;11:1004-1016. 
21. Tavor S, Petit I, Porozov S, et al. CXCR4 regulates migration and development of human acute myelogenous leukemia stem cells in transplanted NOD/SCID mice. Cancer Res 2004;64:2817-2824.

22. Liesveld JL, Bechelli J, Rosell K, et al. Effects of AMD3100 on transmigration and survival of acute myelogenous leukemia cells. Leuk Res 2007;31:1553-1563.

23. Tavor S, Eisenbach M, Jacob-Hirsch J, et al. The CXCR4 antagonist AMD310o impairs survival of human AML cells and induces their differentiation. Leukemia 2008;22:21515158.

24. Oishi S, Fujii N. Peptide and peptidomimetic ligands for CXC chemokine receptor 4 (CXCR4). Org Biomol Chem 2012;10:5720-5731.

25. Beider K, Begin M, Abraham M, et al. CXCR4 antagonist ${ }_{4}$ F-benzoyl-TN14003 inhibits leukemia and multiple myeloma tumor growth. Exp Hematol 2011;39:282-292.

26. Cho BS, Zeng Z, Mu H, et al. Antileukemia activity of the novel peptidic CXCR4 antagonist LY2510924 as monotherapy and in combination with chemotherapy. Blood 2015;126:222-232.

27. Kuhne MR, Mulvey T, Belanger B, et al. BMS-936564/ MDX-1338: a fully human anti-CXCR4 antibody induces apoptosis in vitro and shows antitumor activity in vivo in hematologic malignancies. Clin Cancer Res 2013;19:357366.

28. Peng SB, Zhang X, Paul D, et al. Inhibition of CXCR 4 by LY2624587, a fully humanized anti-CXCR4 antibody induces apoptosis of hematologic malignancies. PLoS One 2016;11:e0150585.

29. Pernasetti F, Liu SH, Hallin M, et al. A novel CXCR4 antagonist IgG1 antibody (PF-06747143) for the treatment of hematological malignancies. Blood 2014;124:2311.

30. Zhang Y, Saavedra E, Tang R, et al. Targeting acute myeloid leukemia with a new CXCR4 antagonist $\mathrm{IgG}_{1}$ antibody (PF-06747143)in NOD/SCID mice. Blood 2015;126:1362.

31. Hsieh YT, Jiang E, Pham J, et al. VLA4 blockade in acute myeloid leukemia. Blood 2013;122:3944.

32. Layani-Bazar A, Skornick I, Berrebi A, et al. Redox modulation of adjacent thiols in VLA-4 by AS1or converts myeloid leukemia cells from a drug-resistant to drug-sensitive state. Cancer Res 2014;74:3092-3103.

33. Chien S, Haq SU, Pawlus M, et al. Adhesion of acute myeloid leukemia blasts to E-selectin in the vascular niche enhances their survival by mechanisms such as Wnt activation. Blood 2013;122:61.
34. Jin L, Hope KJ, Zhai Q, Smadja-Joffe F, Dick JE. Targeting of $\mathrm{CD}_{44}$ eradicates human acute myeloid leukemic stem cells. Nat Med 2006;12:1167-1174.

35. Jacamo R, Chen Y, Wang Z, et al. Reciprocal leukemia-stroma VCAM-1/VLA-4-dependent activation of NF-kappaB mediates chemoresistance. Blood 2014;123:2691-2702.

36. Klein RS, Rubin JB. Immune and nervous system CXCL12 and CXCR4: parallel roles in patterning and plasticity. Trends Immunol 2004;25:306-314.

37. Han AR, Lee JY, Kim HJ, Min WS, Park G, Kim SH. A CXCR4 antagonist leads to tumor suppression by activation of immune cells in a leukemia-induced microenvironment. Oncol Rep 2015;34:2880-2888.

38. Hoellenriegel J, Zboralski D, Maasch C, et al. The Spiegelmer NOX-A12, a novel CXCL12 inhibitor, interferes with chronic lymphocytic leukemia cell motility and causes chemosensitization. Blood 2014;123:1032-1039.

39. Vater A, Sahlmann J, Kroger N, et al. Hematopoietic stem and progenitor cell mobilization in mice and humans by a first-in-class mirror-image oligonucleotide inhibitor of CXCL12. Clin Pharmacol Ther 2013;94:150-157.

40. Uy GL, Rettig MP, Motabi IH, et al. A phase 1/2 study of chemosensitization with the CXCR4 antagonist plerixafor in relapsed or refractory acute myeloid leukemia. Blood 2012;119:3917-3924.

41. Greenberg PL, Lee SJ, Advani R, et al. Mitoxantrone, etoposide, and cytarabine with or without valspodar in patients with relapsed or refractory acute myeloid leukemia and high-risk myelodysplastic syndrome: a phase III trial (E2995). J Clin Oncol 2004;22:1078-1086.

42. Uy GL, Avigan D, Cortes JE, et al. Safety and tolerability of plerixafor in combination with cytarabine and daunorubicin in patients with newly diagnosed acute myeloid leukemia: preliminary results from a phase I study. Blood 2011;118:82.

43. Roboz GJ, Scandura JM, Ritchie E, et al. Combining decitabine with plerixafor yields a high response rate in newly diagnosed older patients with AML. Blood 2013;122:621.

44. Andreeff M, Zeng Z, Kelly M, et al. Mobilization and elimination of FLT3-ITD+ acute myelogenous leukemia (AML) stem/progenitor cells by plerixafor, G-CSF, and sorafenib: phase I trial results in relapsed/refractory AML patients. J Clin Oncol 2014;32(15 Suppl):7033.

45. Borthakur G, Ofran Y, Nagler A, et al. The peptidic CXCR4 antagonist, BL-8040, significantly reduces bone marrow immature leukemia progenitors by inducing differen- 
tiation, apoptosis and mobilization: results of the dose escalation clinical trial in acute myeloid leukemia. Blood 2015;126:2546.

46. Becker PS, Foran JM, Altman JK, et al. Targeting the CXCR4 pathway: safety, tolerability and clinical activity of ulocuplumab (BMS-936564), an anti-CXCR4 antibody, in relapsed/refractory acute myeloid leukemia. Blood 2014;124:386.

47. Rashidi A, DiPersio JF. Targeting the leukemia-stroma interaction in acute myeloid leukemia: rationale and latest evidence. Ther Adv Hematol 2016;7:40-51. 